\title{
Prediction of the Passive Intestinal Absorption of Medicinal Plant Extract Constituents with the Parallel Artificial Membrane Permeability Assay (PAMPA)
}

Authors

Affiliations
Charlotte Petit ${ }^{1}$, Alban Bujard ${ }^{1}$, Krystyna Skalicka-Woźniak ${ }^{2}$, Sylvian Cretton ${ }^{1}$, Joëlle Houriet ${ }^{1}$, Philippe Christen ${ }^{1}$, Pierre-Alain Carrupt ${ }^{1}$, Jean-Luc Wolfender ${ }^{1}$

1 School of Pharmaceutical Sciences, EPGL, University of Geneva, University of Lausanne, Geneva, Switzerland

2 Department of Pharmacognosy with Medicinal Plant Unit, Medical University of Lublin, Lublin, Poland

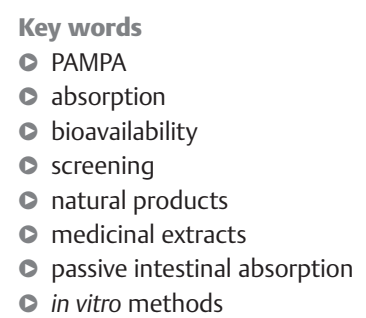

received August 17, 2015 revised Nov. 6, 2015 accepted Dec. 13, 2015

\section{Bibliography}

DOI http://dx.doi.org/ 10.1055/s-0042-101247 Published online February 12 , 2016

Planta Med 2016; 82: 424-431 (c) Georg Thieme Verlag KG Stuttgart · New York . ISSN 0032-0943

\section{Correspondence}

Prof. Dr. Jean-Luc Wolfender School of Pharmaceutical Sciences

Phytochemistry and Bioactive Natural Products

\section{EPGL}

University of Geneva

University of Lausanne

Quai Ernest-Ansermet 30

1211 Geneva 4

Switzerland

Phone: + 41223793400

Fax: +41223793399

jean-luc.wolfender@unige.ch

\section{Abstract \\ $\nabla$}

At the early drug discovery stage, the highthroughput parallel artificial membrane permeability assay is one of the most frequently used in vitro models to predict transcellular passive absorption. While thousands of new chemical entities have been screened with the parallel artificial membrane permeability assay, in general, permeation properties of natural products have been scarcely evaluated. In this study, the parallel artificial membrane permeability assay through a hexadecane membrane was used to predict the passive intestinal absorption of a representative set of frequently occurring natural products. Since natural products are usually ingested for medicinal use as components of complex extracts in traditional herbal preparations or as phytopharmaceuticals, the applicability of such an assay to study the constituents directly in medicinal crude plant extracts was further investigated. Three representative crude plant extracts with different natural product compositions were chosen for this study. The first extract was composed of furanocoumarins (Angelica archangelica), the second extract included alkaloids (Waltheria indica), and the third extract contained flavonoid glycosides (Pueraria montana var. lobata). For each medicinal plant, the effective passive permeability values $\mathrm{P}_{\mathrm{e}}(\mathrm{cm} / \mathrm{s})$ of the main natural products of interest were rapidly calculated thanks to a generic ultrahigh-pressure liquid chromatographyUV detection method and because $\mathrm{P}_{\mathrm{e}}$ calculations do not require knowing precisely the concentra-

\section{Introduction \\ $\nabla$}

In NP research, compounds are isolated from various complex biological matrices (e.g., plant, marine organism, or microbial extracts). The inherent chemical diversity of NPs has resulted in a tion of each natural product within the extracts. The original parallel artificial membrane permeability assay through a hexadecane membrane was found to keep its predictive power when applied to constituents directly in crude plant extracts provided that higher quantities of the extract were initially loaded in the assay in order to ensure suitable detection of the individual constituents of the extracts. Such an approach is thus valuable for the high-throughput, cost-effective, and early evaluation of passive intestinal absorption of active principles in medicinal plants. In phytochemical studies, obtaining effective passive permeability values of pharmacologically active natural products is important to predict if natural products showing interesting activities in vitro may have a chance to reach their target in vivo.

\section{Abbreviations}

$\checkmark$

BBB: blood brain barrier

FA: fraction absorbed

GIT: gastrointestinal tract

HDM: $\quad$ hexadecane membrane

HTS: high-throughput screening

NP: $\quad$ natural product

PAMPA: parallel artificial membrane permeability assay

UHPLC: ultrahigh-pressure liquid chromatography

Supporting information available online at http://www.thieme-connect.de/products

key role of these metabolites and their derivatives in modern drug discovery [1,2]. At the early drug discovery stage, various in vitro screening assays are either applied to crude natural extracts, to fractions, or to pure NPs in order to identify the most promising drug candidates [3]. Usually, this 
large variety of advanced in vitro pharmacological assays is performed before information regarding absorption, distribution, metabolism, excretion, and toxicity is available [4]. However, in the drug development stage, inappropriate pharmacokinetic properties have been recognized to be the primary cause of the withdrawal of new molecules [5]. In current industrial practise, a new effort is being made by integrating the pharmacokinetic profiling in parallel with the pharmacological screening to maximise the chances for selecting superior drug candidates [6]. In this context, a variety of highly accurate and HTS techniques has been developed for the early evaluation of absorption, distribution, metabolism, excretion, and toxicity properties of compounds of interest [7].

For the early prediction of absorption, PAMPA is one of the most frequently used in vitro models $[5,8]$. PAMPA is a simple and robust method to predict the transcellular passive absorption through biological membranes $[9,10]$. The PAMPA measures the ability of a compound to diffuse from a donor compartment (containing the tested compound diluted in buffer) to an acceptor compartment (containing simply buffer), which are separated by a lipophilic artificial membrane and is performed in a 96-well plate. Depending on the composition of the artificial membrane used, different biological barriers can be mimicked, such as the GIT $[11,12]$, the BBB $[13,14]$, or the dermal layer $[15,16]$. After the incubation time, the ratio of compound which has crossed the artificial membrane is calculated and used to obtain the effective passive permeability value $P_{e}(\mathrm{~cm} / \mathrm{s})$, which is a constant value for each compound tested (see Material and Methods for details on the equations). Such an evaluation of the physicochemical properties of a compound of interest is thus very advantageous for the early prediction of its passive absorption because several correlations have been reported in the literature between $\mathrm{P}_{\mathrm{e}}$ values and in vitro or in vivo absorption data $[12,15,17,18]$. The popularity of PAMPA has rapidly risen in the industry as a versatile and cost-effective assay compared to standard cellular models such as Caco-2 $[19,20]$. Other applications of PAMPA such as the prediction of protein binding, lipophilicity determination, and $\mathrm{pH}$ profiling have also been reported $[10,12,21,22]$.

Despite the rapid industrial success of PAMPA in the last decade, papers mentioning the use of this HTS technique for studying the passive permeability potential of NPs are sparse [23-27]. The applicability of a modified version of the PAMPA-BBB technique was recently reported in the frame of a CNS-based HTS campaign in order to screen a library of chemically diverse plant extracts [18]. In the present study, the commonly used and validated method HDM-PAMPA was chosen to investigate the passive absorption through the GIT. In the original paper, the $\log \mathrm{P}_{\mathrm{e}}$ values obtained from such an assay were proven to be highly correlated to the reported human intestinal absorption of common drugs that are known to be passively absorbed (FA) [12]. In this study, the validated HDM-PAMPA was used to obtain the effective passive permeability values $\log \mathrm{P}_{\mathrm{e}}$ of various secondary metabolites from medicinal plants. The first aim of this work was to explore the general passive intestinal absorption of a representative set of pure standards because information about the oral bioavailability of NPs in general is lacking. Since NPs are mainly ingested in the current diet (food, herbal teas) [28] or as medicinal preparations (decoctions, tinctures, or standardised extracts) [29-31], there was also a great interest in studying medicinal plants extracts. The second aim of this work was to evaluate if the HDM-PAMPA kept its predictive power when applied to the study of constituents directly in crude plants extracts.
For this investigation, three representative medicinal plants of very different compositions were selected. Angelica archangelica (L.) H.Karst. (Apiaceae) is used in traditional medicine and reputed to be effective for the treatment of alimentary tract disorders. It is also used as a part of the well-known herbal preparation STW5 (Iberogast ${ }^{\circledR}$ ) - an established gastrointestinal phytotherapeutic medication used to successfully treat functional dyspepsia [32]. Waltheria indica L. (Malvaceae), a short-lived shrub widespread in subtropical and tropical regions of the world, is traditionally used to treat skin ulcers, rheumatism, diarrhea, hemorrhoids, asthma, tooth infections, and even malaria [33, 34]. Pueraria montana var. lobata (Willd.) Sanjappa \& Pradeep (Fabaceae) is a perennial vine native from the South East Asia regions and known as Kudzu in traditional Japanese medicine or Yege in traditional Chinese medicine. Diverse medicinal uses of the roots of $P$. montana var. lobata are reported in the Chinese Pharmacopeia. The plant is used as a general medicine for improving body functions but also more specifically in the treatment of diarrhea, acute dysentery, deafness, or cardiovascular diseases [35]. For each of these selected medicinal plants, a crude plant extract containing the metabolites that are ingested when the medicinal plant is orally taken was prepared. In this paper, the applicability of the original HDM-PAMPA for the study of constituents directly in such extracts with various NPs composition was investigated.

\section{Results and Discussion}

\section{$\nabla$}

In a first phase, a representative set of frequently occurring plant secondary metabolites was studied with the original and validated HDM-PAMPA method in order to have an estimation of their passive intestinal absorption. For this step, the originally used 96-well plate UV reader detection method could have been used [12]. The more sensitive and less variable UHPLC-UV detection method was, however, preferred in order to analyse both pure NPs and complex natural extracts with the same approach. The replacement of the direct UV measurement by LC-UV had, nevertheless, no impact on the permeability results of the PAMPA technique itself and has already been validated [36]. Here, a UHPLC-UV detection method was optimised in order to be generic, fast, and to be directly compatible for the analysis of 96well plates obtained from the HDM-PAMPA allowing the keeping of the high-throughput nature of the assay $[37,38]$. After incubation, the effective passive permeability values $\mathrm{P}_{\mathrm{e}}$ of NPs of interest, either assessed as pure compounds or as constituents of crude plant extracts, were calculated. By referring to the original validated HDM-PAMPA, a $\log \mathrm{P}_{\mathrm{e}}$ value of -4.70 corresponded to an FA of $30 \%$ [12]. This value was chosen for this study as a boundary line between compounds with high potential for passive intestinal absorption $\left(\log \mathrm{P}_{\mathrm{e}} \geq-4.70\right.$ corresponding to a "GIT +" classification) and those showing no significant passive permeation ( $\log \mathrm{P}_{\mathrm{e}} \leq-4.70$ corresponding to a "GIT -" classification). A predicted FA of $30 \%$ is indeed considered an acceptable starting point in many drug discovery programs [20]. For the very low-permeant NPs, the concentration in the acceptor side fell below the limit of detection even if these compounds were satisfactorily detected in the donor compartment. For such low permeant compounds (GIT -), an arbitrary cutoff value was assigned $\left(\log \mathrm{P}_{\mathrm{e}}=-5.52\right)$, which corresponds to an amount of compound reaching the acceptor compartment lower than $1 \%$ after $5 \mathrm{~h}$. 
Using this approach, 49 NPs of various structural types were studied. In order to avoid the need of a compound-dependent detection method, only NPs with a UV chromophore and an appropriate solubility were selected. The effective passive permeability values $\mathrm{P}_{\mathrm{e}}$ of flavonoid aglycones and glycosides, alkaloids, coumarins, and NPs bearing a carboxylic acid function were thus obtained. The results are represented in 0 Fig. 1 according to the structural type of the NPs investigated (grouped by class of NPs). Details on the chemical structure, the effective passive permeability value $\log \mathrm{P}_{\mathrm{e}}$, and the main calculated physicochemical properties of each of the individual NPs tested are available as Supporting Information (Figs. S1-S5 and Table S1, Supporting Information). Amongst the different NPs investigated, a general trend in passive permeability was observed for each class of NPs. The great majority of the coumarins and alkaloids showed good potential for passive permeation (GIT +), while all flavonoid glycosides and acidic NPs were predicted as low permeant compounds (GIT -). For the specific case of the flavonoid aglycones, these NPs displayed various effective passive permeability values. Those being partially methoxylated showed, in general, better permeability properties compared to the fully hydroxylated ones. The high predicted FA of coumarins could be explained since such NPs are small and mildly lipophilic NPs. Such physicochemical properties are favourable for transcellular passive absorption through biological barriers [6]. Aesculetin and scopoletin, which contain one or two hydroxyls, were observed to be less permeant than the other coumarins possessing a methoxy group or having no hydroxy group at all.

The majority of the alkaloids tested were predicted as good candidates for passive intestinal absorption. Since alkaloids are Ncontaining molecules, they can be potentially ionised at physiological pHs. The ionisation state of a compound can be a limiting factor for transcellular passive absorption through membranes. It is indeed commonly accepted that the ionised form of a compound has at least 1000 -fold lower membrane permeability than its corresponding unionised form [12]. Despite the potential ionisation of alkaloids, medium to high permeation values were, however, measured for most of the alkaloids tested. These results illustrated that the transcellular passive permeability through biological membranes could not be explained by a single physicochemical descriptor (e.g., ionisation state pKa, lipophilicity log P, molecular weight Mw, etc.; see Table S1, Supportig Information) and also highlighted the importance of an experimental evaluation of absorption. The high permeation of alkaloids was expected since several of them are known for their CNS activities and are therefore crossing the even more selective BBB biological barrier.

Both flavonoid aglycones and glycosides were tested. All the glycosides tested ( $\mathrm{O}$ - and $\mathrm{C}$-glycosides, occurring as mono or disaccharides) were found to have very low permeability. The presence of sugar moieties increases the molecular weight and the polarity of such compounds, which might limit the passive intestinal absorption. In general, the bioavailability of flavonoid glycosides has been extensively studied since they are ubiquitous micronutrients in various diets $[39,40]$. It has been reported that deglycosylation of such compounds by $\beta$-glucosidase in the small intestine and by the colonic microflora is often required before absorption can occur $[41,42]$. The flavonoids aglycones, either occurring as such in plants or resulting from hydrolysis, displayed various permeability behaviours. Some flavonoid aglycones, in particular the ones containing one or several methoxy groups, were able to cross the HDM barrier: $\log \mathrm{P}_{\mathrm{e}}=-4.91$ for hesperetin,

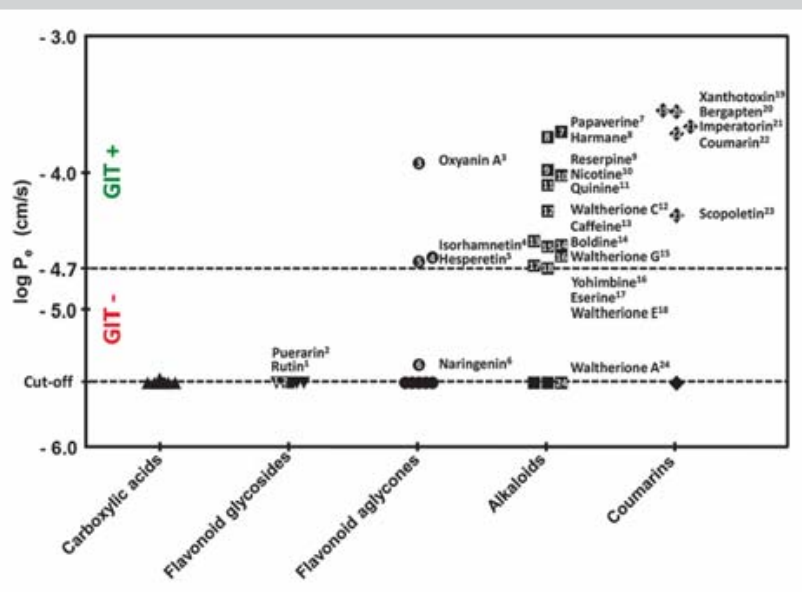

Fig. 1 Effective passive permeability values of all the 49 NPs studied, grouped by main classes of NPs. The dotted line delimits compounds with good predicted passive intestinal absorption $\left(\log P_{e} \geq-4.70=F A \geq 30 \%=\right.$ GIT +) and low-permeability NPs ( $\log P_{\mathrm{e}} \leq-4.70=$ FA $\leq 30 \%=$ GIT -). For the very low-permeability NPs, a cutoff value of $\log P_{e}=-5.52$ was arbitrarily applied. Such compounds were not labelled for clarity reasons, but details on the effective passive permeability values of all the NPs tested are available in Table S1, Supporting Information. (Color figure available online only.)

$\log \mathrm{P}_{\mathrm{e}}=-4.64$ for isorhamnetin and $\log \mathrm{P}_{\mathrm{e}}=-3.92$ for oxyanin $\mathrm{A}$. The fully hydroxylated aglycones were not predicted as good permeants, even those having only few hydroxy groups such as daidzein and naringenin.

Finally, NPs bearing a carboxylic function such as chlorogenic acid, vanillic acid, or sinapic acid fell into the low absorption category (predicted FA $\leq 30 \%$ ). In the experimental conditions used, all passive permeability measurements were performed at pH 6.8, which is a recommended mean value for the FA prediction in the HDM-PAMPA assay [12]. The presence of a carboxylic acid group, which is fully deprotonated at such an experimental $\mathrm{pH}$ and which increases the polarity of such small molecules, might limit the transcellular passive absorption of such acidic NPs.

In a second phase, the HDM-PAMPA method was used to study the passive intestinal absorption of constituents directly in crude plant extracts. For this study, extracts of three medicinal plants containing very different constituents ( $W$. indica, $P$. montana var. lobata, and $A$. archangelica) were selected. The extracts were prepared in order to insure that they contain the metabolites that are ingested when the medicinal plant is orally taken: a methanolic extract of the fruits from $A$. archangelica mostly composed of furanocoumarins $[43,44]$, a decoction of aerial parts of $W$. indica containing quinoline alkaloids [33], and a decoction of roots of $P$. montana var. lobata with flavonoid glycosides [35]. The HDMPAMPA method used for pure NPs was adapted to the analysis of crude plant extracts by simply increasing the amount of sample (crude extract) initially loaded in the donor compartment since single metabolites are found at lower concentrations in mixtures. In the literature, such an increase of the initial loading in the PAMPA has been reported in order to address issues encountered with weak UV visible or poorly soluble pure compounds and has therefore been validated [36]. Contrary to the previous study on pure NPs, the concentrations of individual constituents in the extract could not be fixed because metabolites occur at different 
levels in extracts. The PAMPA method does not, however, require knowing the exact concentration of a compound for its $\mathrm{P}_{\mathrm{e}}$ calculation (see equations in Materials and Methods). Furthermore, the simultaneous detection of the different constituents in a single crude extract necessitates the use of an appropriate detection method. The previously described UHPLC-UV method was adapted to provide a higher peak capacity and to insure satisfactory separation for direct comparison of the UV peaks of compounds of interest. This method was also optimised to be generic and fast (UHPLC run of $5 \mathrm{~min}$ ) in order to keep the original highthroughput nature of the PAMPA assay.

At first, the robustness of the HDM-PAMPA/UHPLC-UV method for the direct assessment of crude extracts was evaluated. Five standards with known $\mathrm{P}_{\mathrm{e}}$ values were selected and spiked into the three different crude plant extract matrices. The resulted spiked extracts were directly assessed in the HDM-PAMPA and the effective passive permeability values of each of the five standards were calculated in the different conditions (Table S2, Supporting Information). The aim was to check if their individual effective passive permeability values were not affected when measured in complex mixtures. The artificial mixture was made of two synthetic compounds recognised as permeability standards for HDM-PAMPA (testosterone and piroxicam) [12] and three representative NP standards analysed above (rutin, coumarin, and hesperetin). The standards mixture was first assessed in the HDM-PAMPA and the adapted and generic UHPLC-UV detection method was used. The passive permeability results are illustrated in 0 Fig. $2 \mathrm{~A}$ where the UHPLC chromatograms of both compartments are compared (donor compartment at initial time and acceptor compartment after incubation). The calculated effective passive permeability values $\left(\log \mathrm{P}_{e}\right)$ of the five standards either assessed as a mixture or as pure compounds were compared (Table S2, Supporting Information) and were found not to be affected, as illustrated in $\mathbf{O}$ Fig. $\mathbf{3} \mathbf{A}$. The same standard mixture was then spiked into the three selected crude plant extracts and these resulting spiked extracts were assessed in the HDM-PAMPA ( $\odot$ Fig. 2B1, C1, D1). The $\log \mathrm{P}_{\mathrm{e}}$ values of each five standards were calculated in the three conditions (Table S2) and were compared to $\log \mathrm{P}_{\mathrm{e}}$ values obtained when assessing pure standards individually. As for the standard mixture alone, and as illustrated in - Fig. 3B-D, no significant differences between the different spiked extracts were observed. These experiments indicate that the individual permeability of standards is not affected by the presence of plant matrix constituents in the donor compartment and thus that the method has a good potential to correctly estimate individual effective passive permeability values of any constituents of an extract provided that they are well resolved and well detected by the UHPLC-UV detection.

Finally, the passive intestinal absorption of the constituents of interest within the three selected medicinal plant extracts was further investigated with HDM-PAMPA. For the A. archangelica and $P$. montana var. lobata extracts, the generic UHPLC-UV gradient method previously used for the analysis of the five standards mixture provided sufficient peak capacity for a satisfactory profiling of the main secondary metabolites of interest in the extracts. For the $W$. indica extract, the UHPLC gradient had to be optimised in order to obtain a better resolution for the $\mathrm{P}_{\mathrm{e}}$ calculation.

The passive permeability results obtained from the HDM-PAMPA analysis of the three crude extracts are illustrated in $\mathbf{O}$ Fig. 2 B2, C2, D2. For the A. archangelica methanolic extract, most of the compounds eluting between $2.0 \mathrm{~min}$ and $3.0 \mathrm{~min}$ were detected

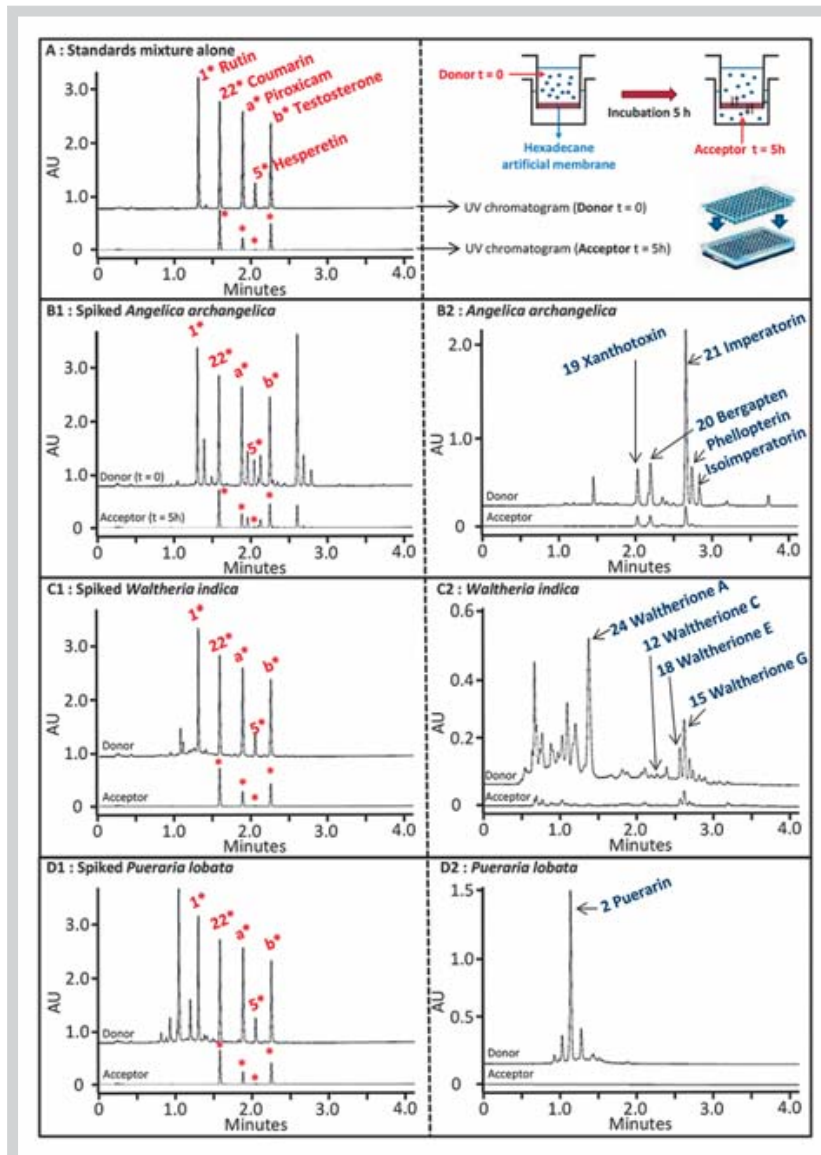

Fig. 2 Comparison of the UHPLC-UV chromatograms prior (donor at $\mathrm{t}=0 \mathrm{~h}$ ) and after the HDM-PAMPA experiment (acceptor at $\mathrm{t}=5 \mathrm{~h}$ ). UV traces are represented at $254 \mathrm{~nm}$. A Standards mixture alone (the five standards are indicated with an asterisk). B1 The $A$. archangelica methanolic extract spiked with the standards; $C 1$ the $W$. indica decoction spiked with the standards; and $\mathbf{D} 1$ the $P$. montana var. lobata ethanolic extract spiked with the standards. For crude plant extracts alone, UV traces are represented at $300 \mathrm{~nm}$. B2 The $A$. archangelica extract; $\mathbf{C} 2$ the $W$. indica; and $\mathbf{D} 2$ the $P$. montana var. lobata extract. Details on the effective passive permeability values calculated are available in Table S2, Supporting Information. (Color figure available online only.)

in the acceptor compartment ( $\odot$ Fig. 2 B2), indicating that several constituents in the extract had a good potential for passive absorption. These moderate to high permeant compounds were identified as furanocoumarins by comparing their retention times, UV-DAD, and mass spectra with standards analysed under the same conditions as well as by relating to the MS data from the literature [45-47]. Their effective passive permeability values were simply calculated by integrating their resolved UV peaks in the extracts without the need for information on their concentrations (Table S2, Supporting Information). These permeability results indicate that the furanocoumarins in A. archangelica have good potential for easily crossing the gastrointestinal barrier via the transcellular route. The three main constituents of the extracts (imperatorin, bergapten, and xanthotoxin) were also assessed in the previous series of measurements made on pure NPs. Similar to the above study of standards mixtures, the individual effective passive permeability values of these furanocoumarins were found to be similar to the values obtained with the A. archangelica extract (see above). 

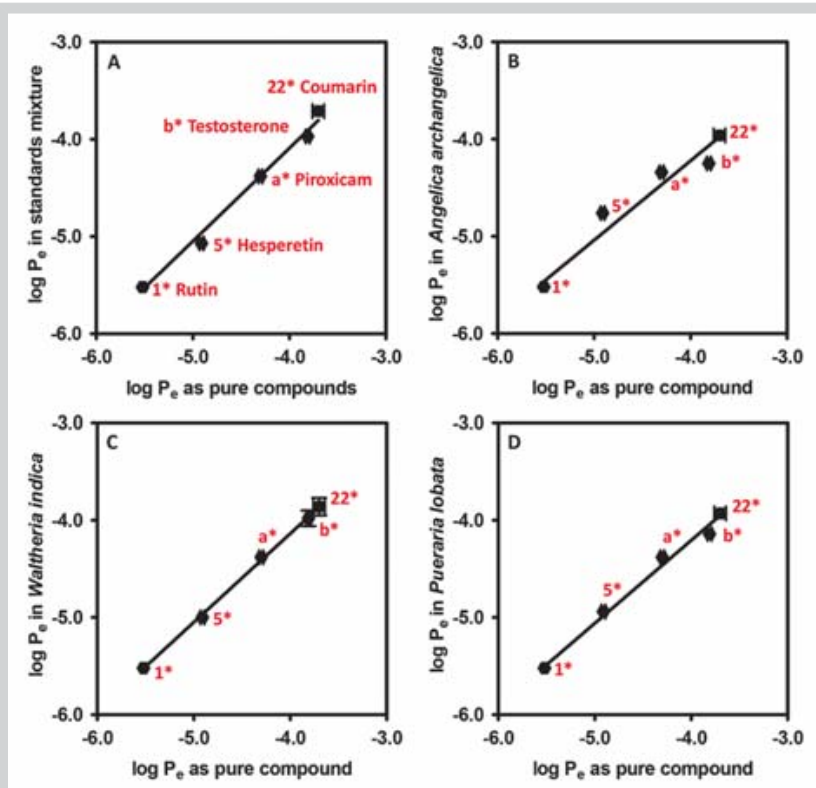

Fig. 3 Comparison between the effective passive permeability values of the five standards, either obtained when assessing each standard individually or when assessing the mixtures in the HDM-PAMPA. The spiked standards are distinguished by an asterisk, as in $\bullet$ Fig. 2 . The plain line represents the linear regression between the permeability results. A Standards mixture alone: $\log P_{e}=0.96( \pm 0.04) \times \log P_{e, p u r e}-0.26( \pm 0.17) ; r^{2}=0.993$. B The $A$. archangelica methanolic extract spiked with the standards: log $P_{\mathrm{e}}=0.82( \pm 0.07) \times \log P_{e, p u r e}-0.95( \pm 0.34) ; r^{2}=0.963$. (C) The W. indica decoction spiked with the standards: $\log P_{e}=0.91( \pm 0.01) \times \log P_{e, p u r e}{ }^{-}$ $0.49( \pm 0.05) ; r^{2}=0.999$. D The $P$. montana var. lobata ethanolic extract spiked with the standards: $\log \mathrm{P}_{\mathrm{e}}=0.85( \pm 0.03) \times \log \mathrm{P}_{\mathrm{e}, \text { pure }}-0.79$ $( \pm 0.15) ; r^{2}=0.994$. Confidence intervals $(95 \%)$ are given in brackets. A high coefficient of determination in each different case was observed, over a wide range of permeability values. Details on the effective passive permeability values are available in Table S2, Supporting Information. (Color figure available online only.)

For the $W$. indica extract, compounds of different polarities, two groups (retention times from $0.6 \mathrm{~min}$ to $1.3 \mathrm{~min}$ and from $2.2 \mathrm{~min}$ to $3.8 \mathrm{~min}$ ) were found in the acceptor compartment ( $\bullet$ Fig. 2 C2). A complementary LC-MS-based dereplication indicated that the more lipophilic NPs belong to the quinoline alkaloids waltherione C, E, and G and to flavonoid aglycones, whereas the more polar group of constituents included cyclopeptide alkaloids adouetin $X$ and $Y[33,34]$. The effective passive permeability values of the pharmacologically active waltheriones C, E, and G were calculated and such compounds were found to have medium permeability ( Fig. 1 and Table S2, Supporting Information). The chromatographic resolution of the most polar constituents did not allow a precise permeability assessment in the HTS conditions used. However, one of the main constituents detected in the UV trace (RT $1.4 \mathrm{~min}$ in the donor compartment) was found to have very low permeability and was dereplicated as waltherione A (๑ Fig. 1 and Table S2, Supporting Information). Compared to the medium permeant waltherione $\mathrm{C}$, waltherione $\mathrm{A}$ bears an additional hydroxy group in the cycloheptane ring (Fig. S1, Supporting Information) [34].

The crude extract of $P$. montana var. lobata contains several flavonoid glycosides, including the main constituent and pharmacologically active flavonoid C- glycoside puerarin [35]. None of the main constituents of the extract was able to cross the HDM bar- rier ( Fig. 2D2), indicating that a transcellular passive transport of the flavonoid glycosides in P. montana var. lobata is unlikely. Such results are consistent with our previous study on the passive permeation of both individual O- and C- flavonoid glycosides and are also in good agreement with in vivo studies relating the essential role of active transport of puerarin for its intestinal absorption $[48,49]$.

The permeability measurements performed on these three very different extracts have shown the practical applicability of the HDM-PAMPA to such complex samples. Clear differences in passive permeability could be evidenced for the different constituents of the extracts. Such dissimilarities between these profiled metabolites could not simply be based on their polarity (such as their retention times $[50,51])$. These findings have also indicated that the presence of multiple component mixtures did not influence the passive permeability of single constituents and thus that the passive absorption of NPs seemed not to be potentially influenced by synergistic aspects in the frame of the HDM-PAMPA predictions.

Using such an approach to predict the passive intestinal absorption of NPs within medicinal plant extracts is highly valuable since NPs are, in general, ingested as constituents of standardised extracts in phytopharmaceuticals or as simple decoctions for most medicinal plants. Obtaining the effective passive permeability values of NPs directly in extracts is also worthy at the early drug discovery stage in order to prioritize the isolation of bioactive compounds that have better chances to reach their target in vivo and to verify if bioactive principles of known medicinal plants have a good potential to be passively absorbed.

The method presented here suffers, however, from some limitations. On the one hand, non-UV detectable and very low soluble NPs can be difficult to analyse. For these specific issues, highly sensitive MS/MS detection methods can be adapted in order to analyse the PAMPA compartments using the same approach [52, 53]. In this case, however, the analytical method would be compound-dependent and less adapted to a high-throughput and generic screening. On the other hand, the evaluation of permeability cannot be only restricted to passive absorption mechanisms, even if the passive transport is widely recognized to be the preferred route for intestinal absorption [8]. An investigation of other routes of transport such as the active transport could become worth considering for low passive permeability compounds such as glycosides. For such deeper investigations, alternative methods such as cell-based assays could be worth considering [54]. They, however, do not provide the same cost-effectiveness, robustness, and ease of usage as the PAMPA $[5,17]$.

\section{Materials and Methods}

$\nabla$

\section{Chemicals}

Bergapten ( $\geq 99 \%)$, caffeic acid ( $\geq 97 \%)$, caffeine ( $\geq 99.0 \%)$, colchicine $(\geq 95 \%)$, epicatechin ( $\geq 97 \%)$, harmane ( $\geq 98 \%)$, 4-hydroxyphenylacetic acid ( $\geq 98 \%$ ), imperatorin ( $\geq 98 \%$ ), nicotinic acid ( $\geq 98 \%$ ), naringin ( $\geq 95 \%)$, quercitrin $(\geq 95 \%)$, papaverine hydrochloride ( $\geq 98 \%$ ), piroxicam ( $\geq 97 \%$ ), sulfasalazine ( $\geq 98 \%$ ), theophylline $(\geq 99 \%)$, trans-cinnamic acid ( $\geq 97 \%)$, xanthotoxin ( $\geq 98 \%$ ), yohimbine hydrochloride ( $\geq 98 \%$ ), hexadecane $(\geq 99 \%)$, anhydrous disodium hydrogen phosphate ( $\geq 99.0 \%$ ), and potassium dihydrogen phosphate ( $\geq 99.0 \%$ ) were purchased from Sigma. Aesculetin ( $\geq 98 \%)$, chlorogenic acid ( $\geq 95 \%)$, ferulic acid $(\geq 98 \%)$, hyperoside ( $\geq 99 \%$ ), isoquercitrin ( $\geq 80 \%$ ), luteolin-7-O-glucoside 
( $\geq 98 \%$ ), morin dihydrate ( $\geq 80 \%)$, naringenin ( $\geq 95 \%)$, rutin trihydrate $(\geq 99 \%)$, scopoletin ( $\geq 98 \%$ ), and vitexin-2-O-rhamnoside ( $\geq 80 \%$ ) were purchased from Carl Roth $\mathrm{GmbH}$. Eserine ( $\geq 98.0 \%)$, hesperetin ( $\geq 99 \%$ ), nicotine ( $\geq 99 \%$ ), quinine ( $\geq 90 \%$ ), salicylic acid ( $\geq 99 \%$ ), sinapic acid ( $\geq 98.0 \%$ ), syringic acid ( $\geq 97.0 \%$ ), testosterone $(\geq 99 \%)$, and vanillic acid $(\geq 97.0 \%)$ were purchased from Fluka Chemie AG. Coumarin ( $\geq 98 \%$ ), daidzein ( $\geq 98 \%$ ), and potassium chloride $(\geq 99 \%)$ were purchased from Acros. DMSO $(\geq 99.7 \%)$ was purchased from Fischer Chemical. Hexane ( $\geq 97 \%)$ was purchased from VWR. Puerarin ( $\geq 98 \%$ ) was purchased from Chengdu Biopurity Phytochemicals. Phellopterin $(\geq 99 \%)$ and isoimperatorin $(\geq 98 \%$ ) was purchased from PhytoLab $\mathrm{GmbH}$. Waltherione A, C, E, and G were isolated and dereplicated from $W$. indica as previously described (minimum purity of $95 \%$ ) [33, 34].

\section{Plant materials and extractions}

The fruits of $A$. archangelica were collected in August 2012 in Medicinal Plant Garden, Medical University of Lublin, Poland, and identified by Krystyna Dąbrowska (Botanical Garden of Maria Curie-Skłodowska University, Lublin). A voucher specimen (KSWAA2012) is deposited at the Department of Pharmacognosy, Medical University of Lublin, Poland. Air-dried and powdered fruits $(50 \mathrm{~g})$ were extracted under reflux with methanol ( $3 \times 700 \mathrm{~mL}, 30 \mathrm{~min}$ each). After filtration, the obtained filtrates were combined together and evaporated under vacuum $(6.12 \mathrm{~g})$. The aerial parts of $W$. indica were collected in February 2013 in Zinder (Niger). Their identification was confirmed by Didier Roguet (Botanical Garden of Geneva). Voucher specimens are deposited at the Botanical Garden of Geneva (no. 19003). Air-dried aerial parts $(50 \mathrm{~g})$ were powdered and extracted with $0.5 \mathrm{~L}$ of boiling water for $1 \mathrm{~h}$. After filtration, the aqueous extract was evaporated and concentrated to $100 \mathrm{~mL}$. Twenty milliliters were loaded onto an Oasis HLB SPE column (Waters) previously activated with $\mathrm{MeOH}$. A wash step with $100 \%$ water was performed in order to remove highly polar compounds. The compounds of interest were eluted with a mixture of $\mathrm{MeOH} / \mathrm{H}_{2} \mathrm{O}(85 / 15)$ and the recovered filtrate was evaporated to dryness $(20 \mathrm{mg})$.

The dry roots of $P$. montana var. lobata were purchased from RatsApotheke (Stralsund, Germany). Liquid nitrogen was added onto the dried roots of P. montana var. lobata to be grounded. Two hundred grams of powdered material were extracted in two liters of boiling water under stirring for $40 \mathrm{~min}$. After filtration at $60^{\circ} \mathrm{C}$, the filtrates were lyophilized until complete drying $(100 \mathrm{~g})$.

\section{Spiking experiments}

Before the HDM-PAMPA experiments, all samples were prepared in pure DMSO (stock solutions) and stored at $4{ }^{\circ} \mathrm{C}$. Stock solutions were prepared at $1 \mathrm{mg} / \mathrm{mL}$ for pure NPs or $10 \mathrm{mg} / \mathrm{mL}$ for crude plant extracts. For the standard mixture experiment, stock solutions ten times more concentrated were prepared in DMSO. Using these solutions, the standards mixture was prepared in order to reach a final concentration of $1 \mathrm{mg} / \mathrm{mL}$ of each standard in pure DMSO (testosterone, piroxicam [12], rutin, coumarin, and hesperetin). Similarly, stock solutions for each of the three spiked crude plant extracts were prepared (A. archangelica, $W$. indica, and $P$. montana var. lobata). These solutions were made in order to reach a final concentration of $1 \mathrm{mg} / \mathrm{mL}$ of each standard and $10 \mathrm{mg} / \mathrm{mL}$ of extract.
Parallel artificial membrane permeability assay through hexadecane membrane experiments All stock solutions were prepared in DMSO at $1 \mathrm{mg} / \mathrm{mL}$ for pure NPs or $10 \mathrm{mg} / \mathrm{mL}$ for crude extracts. These stock solutions were then diluted 20 times with a buffer made of $41 \mathrm{mM} \mathrm{Na} 2 \mathrm{HPO}_{4}$ $45 \mathrm{mM} \mathrm{KH}_{2} \mathrm{PO}_{4}$, and $100 \mathrm{mM} \mathrm{KCl}$ (final pH 6.8) to obtain donor solutions at $\mathrm{t}=0$, containing $5 \%$ DMSO. A 96-well microtiter polycarbonate filter plate (Millipore AG) was impregnated with $15 \mu \mathrm{L}$ of a hexadecane/hexane $(5 / 95 \mathrm{v} / \mathrm{v})$ solution using a Precision $2000^{\circledR}$ 96/384 Well Automated Microplate Pipetting System (Bio-Tek Instrument Inc.). The polycarbonate filter specifications included $5-20 \%$ porosity with a $0.45-\mu \mathrm{m}$ pore size and $9-10 \mu \mathrm{m}$ thickness (the maximum porosity value was taken into account for this study). After membrane impregnation with hexadecane/ hexane $(5 / 95 \mathrm{v} / \mathrm{v})$, the filter plate was placed under a fume hood and subjected to constant shaking $\left(75 \mathrm{rpm}\right.$; Titramax $1000^{\circledR}$, Huber \& Co. AG) for approximately $40 \mathrm{~min}$ to evaporate the hexane. Such filter plate constituted the donor plate, which was filled with $280 \mu \mathrm{L}$ of the donor solutions (containing the test compounds). The donor plate was placed upon a Teflon ${ }^{\circledR}$ 96-well acceptor plate (MSSACCEPTOR, Millipore AG), which had been previously filled with $280 \mu \mathrm{L}$ of buffer containing $41 \mathrm{mM}$ $\mathrm{Na}_{2} \mathrm{HPO}_{4}, 45 \mathrm{mM} \mathrm{KH} \mathrm{PO}_{4}$, and 5\% DMSO. The resulting sandwich was protected with Parafilm to prevent evaporation and incubated at room temperature at $75 \mathrm{rpm}$ for $5 \mathrm{~h}$. After incubation $(5 \mathrm{~h})$, the sandwich was dissociated and the donor and acceptor wells were transferred to black 96-well plates (MaxiSorp, Milian SA) and sealed with heat sealing foil (Waters) prior to UHPLC-UV analysis. Two standards were randomly placed in each plate layout in order to validate the effective passive permeability values obtained. The standards were selected from the original publication for their known effective passive permeability values: sulfasalazine, which is known to be a non-permeant compound and testosterone, which is known to be a high permeant compound (Table S1, Supporting Information) [12]. Thus, for each PAMPA plate, the integrity of the artificial membranes was checked using sulfasalazine as a negative control and the validity of the effective passive permeability results was insured by using testosterone as a positive control. Each compound was analysed in triplicate or quadruplicate and the permeability assays were performed on different days. The calculated effective passive permeability values $\mathrm{P}_{\mathrm{e}}$ were retained only if $\mathrm{RSD}<15 \%$ as quality control was fulfilled.

\section{Ultrahigh-pressure liquid chromatography-ultraviolet detection}

UHPLC measurements were performed using an Acquity UPLC system (Waters), which included a binary pumping system, an autosampler, a UV-VIS diode array programmable detector and a column manager over a pre-column heater. The systems were controlled using Empower Software v2.0, and UV detection was performed from 200 to $400 \mathrm{~nm}$ ( $6 \mathrm{~nm}$ resolution). The chromatograms were extracted at the appropriate UV wavelengths for each NP. The injection was performed in full loop mode $(10 \mu \mathrm{L})$, the column temperature was set at $40^{\circ} \mathrm{C}$, and the flow rate was fixed at $0.5 \mathrm{~mL} / \mathrm{min}$. The binary system used two mobile phases: water with $0.1 \%$ formic acid (A) that had been filtered at $0.22 \mu \mathrm{m}$ and HPLC grade acetonitrile with $0.1 \%$ formic acid (B). For the analyses of pure NPs, an Acquity UPLC ${ }^{\circledR}$ BEH Shield RP18 column $(1.7 \mu \mathrm{m}, 2.1 \times 30 \mathrm{~mm}$; Waters) was used. A generic linear gradient was applied from $98 \%$ of $A$ and $2 \%$ of $B$ to $100 \%$ B over a period of $2.00 \mathrm{~min}$, followed by an isocratic step of $100 \%$ of $B$ during 
$0.20 \mathrm{~min}$. The total analysis time was $3.00 \mathrm{~min}$, including a reequilibration step. For the analyses of the standard mixture and UV visible constituents in the crude plant extracts, an Acquity UPLC ${ }^{\circledR}$ Shield RP18 $(1.7 \mu \mathrm{m}, 2.1 \times 50 \mathrm{~mm}$; Waters $)$ was used as the stationary phase. A linear gradient was applied from $98 \%$ of $A$ and $2 \%$ of $B$ to $100 \%$ of $B$ over a period of $4.20 \mathrm{~min}$, followed by an isocratic step during $0.10 \mathrm{~min}$ of $100 \%$ of $\mathrm{B}$. The total analysis time was $5.00 \mathrm{~min}$, including a reequilibration step. For the $W$. indica extract, the same $50 \mathrm{~mm}$ column was used and only the gradient was modified. A linear gradient was applied from $98 \%$ of $A$ and $2 \%$ of $B$ to $72 \%$ of $A$ and $28 \%$ of $B$ over a period of $0.37 \mathrm{~min}$, followed by a linear gradient from 28 to $31 \%$ of B over a period of $1.43 \mathrm{~min}$, then a linear gradient from 31 to $100 \%$ of $B$ over a period of $2.80 \mathrm{~min}$. An isocratic step was then applied during $0.1 \mathrm{~min}$ with $100 \%$ of $\mathrm{B}$. The total analysis time was $5.00 \mathrm{~min}$, including a reequilibration step. Adequate UHPLC/UV data treatments were ensured by checking the UV signals of all the compounds of interest in the donor compartment at the initial time. Only UV areas that had a signal/noise ratio greater than 100 times were considered relevant for accurate $\mathrm{P}_{\mathrm{e}}$ measurements, while a signal of low intensity were considered irrelevant.

\section{Effective passive permeability values calculations}

The effective passive permeability value $\left(\mathrm{P}_{\mathrm{e}}\right)$ is calculated according to the two-way flux equations, obtained from Fick's law [10, 12]:

$\mathrm{P}_{\mathrm{e}}=\frac{-2.303 \mathrm{~V}_{\mathrm{D}}}{\mathrm{A}\left(\mathrm{t}-\tau_{\mathrm{sS}}\right)} \times\left(\frac{1}{1+\mathrm{r}_{\mathrm{v}}}\right) \times \log \left[1-\left(\frac{1+\mathrm{r}_{\mathrm{v}}^{-1}}{(1-\mathrm{R})}\right) \times \frac{\mathrm{C}_{\mathrm{A}}(\mathrm{t})}{\mathrm{C}_{\mathrm{D}}(0)}\right]$

Where $\mathrm{P}_{\mathrm{e}}$ is the effective passive permeability coefficient $(\mathrm{cm} / \mathrm{s})$,

$r_{\mathrm{v}}=\frac{\mathrm{V}_{\mathrm{D}}}{\mathrm{V}_{\mathrm{A}}}$

$\mathrm{V}_{\mathrm{A}, \mathrm{D}}$ is the volume of the acceptor and donor compartment, respectively $\left(0.28 \mathrm{~cm}^{3}\right), A$ is the membrane area calculated with the maximal porosity $\left(0.052 \mathrm{~cm}^{2}\right), t$ is the time of incubation (s), $\tau_{\mathrm{SS}}$ represents the time required to saturate the membrane with the solute (neglected compared to the total incubation time [10]), and $C_{A, D}(t)$ is the concentration at time $t$ in the acceptor and donor compartment, respectively $\left(\mathrm{mol} / \mathrm{cm}^{3}\right)$. $\mathrm{R}$ represents the membrane retention and is defined as follows:

$(1-R) V_{D} C_{D}(0)=V_{D} C_{D}(t)+V_{A} C_{A}(t)$

For $\mathrm{P}_{\mathrm{e}}$ calculations, only the ratio between the concentrations of a compound of interest in the donor and in the acceptor compartment is needed.

\section{Supporting information}

Chemical structures, effective passive permeability values, and calculated physicochemical descriptors of all 49 NPs as well as permeability values of the standards are available as Supporting Information.

\section{Acknowledgements}

$\nabla$

We gratefully acknowledge helpful discussions with Dr. Sophie Martel and Dr. Soura Challal. We are thankful to Dr. Sven Schröder (HanseMerkur Center for Traditional Chinese Medicine at the University Medical Center Hamburg-Eppendorf) for kindly providing the $P$. montana var. lobata dry roots and crude plant extract.

\section{Conflict of Interest}

The authors declare no conflict of interest.

\section{References}

1 Drewry DH, Macarron R. Enhancements of screening collections to address areas of unmet medical need: an industry perspective. Curr Opin Chem Biol 2010; 14: 289-298

2 David B, Wolfender JL, Dias DA. The pharmaceutical industry and natural products: historical status and new trends. Phytochem Rev 2015; 14: 299-315

3 Queiroz EF, Wolfender JL. Innovative strategies in the search for bioactive plant constituents. In: Meyers RA, editor. Encyclopedia of analytical chemistry. Chichester: John Wiley \& Sons, Ltd.; 2006: 1-22

4 Butterweck V, Nahrstedt A. What is the best strategy for preclinical testing of botanicals? A critical perspective. Planta Med 2012; 78: 747-754

5 Balimane PV, Han YH, Chong SH. Current industrial practices of assessing permeability and P-glycoprotein interaction. AAPS J 2006; 8: E1E13

6 Lipinski CA. Drug-like properies and the causes of poor solubility and poor permeability. J Pharmacol Toxicol Methods 2000; 44: 235-249

7 Wang J, Bell L. Technical challenges and recent advances of implementing comprehensive ADMET tools in drug discovery. In: Zhang D, Surapaneni S, editors. ADME-Enabling technologies in drug design and development. Hoboken, New Jersey: John Wiley \& Sons, Inc.; 2012: 129159

8 Sugano K, Kansy M, Artursson P, Avdeef A, Bendels S, Di L, Ecker GF, Faller $B$, Fischer H, Gerebtzoff G, Lennernaes H, Senner F. Coexistence of passive and carrier-mediated processes in drug transport. Nat Rev Drug Discov 2010; 9: 597-614

9 Faller $B$. Artificial membrane assays to assess permeability. Curr Drug Metab 2008; 9: 886-892

10 Avdeef A. Permeability - PAMPA. In: Avdeef A, editor. Absorption and drug development. Solubility, permeability and charge state, 2nd edition. Hoboken, New Jersey: Wiley; 2012: 319-498

11 Kansy M, Senner F, Gubernator K. Physicochemical high throughput screening: parallel artificial membrane permeation assay in the description of passive absorption processes. J Med Chem 1998; 41: $1007-1010$

12 Wohnsland F, Faller B. High-throughput permeability pH profile and high-throughput alkane/water log P with artificial membranes. J Med Chem 2001; 44: 923-930

13 Le Bourdonnec Passeleu C. Prediction of passive blood-brain barrier permability with PAMPA: from small molecules to complex formulations [dissertation]. Geneva: University of Geneva, School of Pharmaceutical Sciences; 2013

14 Di L, Kerns EH, Fan K, McConnell OJ, Carter GT. High throughput artificial membrane permeability assay for blood-brain barrier. Eur J Med Chem 2003; 38: 223-232

15 Ottaviani G, Martel S, Carrupt PA. Parallel artificial membrane permeability assay: a new membrane for the fast prediction of passive human skin permeability. J Med Chem 2006; 49: 3948-3954

16 Sinko B, Garrigues TM, Balogh GT, Nagy ZK, Tsinman O, Avdeef A, TakacsNovak K. Skin-PAMPA: a new method for fast prediction of skin penetration. Eur J Pharm Sci 2012; 45: 698-707

17 Kerns EH, Di L, Petusky S, Farris M, Ley R, Jupp P. Combined application of parallel artificial membrane permeability assay and Caco-2 permeability assays in drug discovery. J Pharm Sci 2004; 93: 1440-1453

18 Könczöl A, Müller J, Földes E, Béni Z, Végh K, Kéry A, Balogh GT. Applicability of a blood-brain barrier specific artificial membrane permeability assay at the early stage of natural product-based CNS drug discovery. J Nat Prod 2013; 76: 655-663

19 Avdeef $A$. The rise of PAMPA. Expert Opin Drug Metab Toxicol 2005; 1: 325-342

20 Matsson P, Bergström CA, Nagahara N, Tavelin S, Norinder U, Artursson P. Exploring the role of different drug transport routes in permeability screening. J Med Chem 2005; 48: 604-613 
21 Bujard A, Sol M, Carrupt PA, Martel S. Predicting both passive intestinal absorption and the dissociation constant toward albumin using the PAMPA technique. Eur J Pharm Sci 2014; 63: 36-44

22 Bujard A, Voirol H, Carrupt PA, Schappler J. Modification of a PAMPA model to predict passive gastrointestinal absorption and plasma protein binding. Eur J Pharm Sci 2015; 77: 273-278

23 Avdeef A, Strafford M, Block E, Balogh MP, Chambliss W, Khan I. Drug absorption in vitro model: filter-immobilized artificial membranes. Studies of the permeability properties of lactones in Piper methysticum forst. Eur J Pharm Sci 2001; 14: 271-280

24 Venzke D, Carvalho K, Ruani AP, Oliveira AS, Brighente IM, Micke GA, Barison A, Pizzolatti MG. PAMPA permeability, acetylcholinesterase inhibition and antioxidant activity of pyranoisoflavones from Polygala molluginifolia (polygalaceae). J Braz Chem Soc 2013; 24: 1991-1997

25 Rastogi H, Jana S. Evaluation of physicochemical properties and intestinal permeability of six dietary polyphenols in human intestinal colon adenocarcinoma Caco-2 cells. Eur J Drug Metabol Pharmacokinet [Epub ahead of print] 29 Oct 2014; DOI: 10.1007/s13318-014-0234-5

26 Serra H, Mendes T, Bronze MR, Simplicio AL. Prediction of intestinal absorption and metabolism of pharmacologically active flavones and flavanones. Bioorg Med Chem 2008; 16: 4009-4018

27 Tarrago T, Kichik N, Claasen B, Prades R, Teixido M, Giralt E. Baicalin, a prodrug able to reach the CNS, is a prolyl oligopeptidase inhibitor. Bioorg Med Chem 2008; 16: 7516-7524

28 Dillard CJ, German JB. Phytochemicals: nutraceuticals and human health. J Sci Food Agric 2000; 80: 1744-1756

29 Liu JY, Lee KF, Sze CW, Tong Y, Tang SC, Ng TB, Zhang YB. Intestinal absorption and bioavailability of traditional Chinese medicines: a review of recent experimental progress and implication for quality control. J Pharm Pharmacol 2013; 65: 621-633

30 Gertsch J. Botanical drugs, synergy, and network pharmacology: forth and back to intelligent mixtures. Planta Med 2011; 77: 1086-1098

31 Nicoletti $M$. Nutraceuticals and botanicals: overview and perspectives. Int J Food Sci Nutr 2012; 63: 2-6

32 Schemann M, Michel K, Zeller F, Hohenester B, Rühl A. Region-specific effects of STW 5 (Iberogast ${ }^{\circledR}$ ) and its components in gastric fundus, corpus and antrum. Phytomedicine 2006; 13 (Suppl. 5): 90-99

33 Cretton S, Breant L, Pourrez L, Ambuehl C, Marcourt L, Ebrahimi SN, Hamburger M, Perozzo R, Karimou S, Kaiser M, Cuendet M, Christen P. Antitrypanosomal quinoline alkaloids from the roots of Waltheria indica. J Nat Prod 2014; 77: 2304-2311

34 Cretton S, Bréant L, Pourrez L, Ambuehl C, Perozzo R, Marcourt L, Kaiser $M$, Cuendet M, Christen P. Chemical constituents from Waltheria indica exert in vitro activity against Trypanosoma brucei and T. cruzi. Fitoterapia 2015; 105: 55-60

35 Zhang Z, Lam TN, Zuo Z. Radix Puerariae: an overview of its chemistry, pharmacology, pharmacokinetics, and cinical use. J Clin Pharmacol 2013; 53: 787-811

36 Avdeef A, Bendels S, Di L, Faller B, Kansy M, Sugano K, Yamauchi Y. PAMPA - critical factors for better predictions of absorption. J Pharm Sci 2007; 96: 2893-2909

37 Eugster $P$, Wolfender JL. UHPLC in natural products analysis. In: Guillarme D, Veuthey JL, editors. UHPLC in life sciences. Cambridge: Royal Society of Chemistry; 2012: 357-391

38 Eugster P, Guillarme D, Rudaz S, Veuthey JL, Carrupt PA, Wolfender JL. Ultra high pressure liquid chromatography for crude plant extract profiling. J AOAC Int 2011; 94: 51-70

39 Scalbert A, Williamson G. Dietary intake and bioavailability of polyphenols. J Nutr 2000; 130: 2073S-2085S
40 Manach C, Williamson G, Morand C, Scalbert A, Remesy C. Bioavailability and bioefficacy of polyphenols in humans. I. Review of 97 bioavailability studies. Am J Clin Nutr 2005; 81: 230S-242S

41 Passamonti S, Terdoslavich M, Franca R, Vanzo A, Tramer F, Braidot E, Petrussa E, Vianello A. Bioavailability of flavonoids: a review of their membrane transport and the function of bilitranslocase in animal and plant organisms. Curr Drug Metab 2009; 10: 369-394

42 Németh K, Plumb GW, Berrin JG, Juge N, Jacob R, Naim HY, Williamson G, Swallow DM, Kroon PA. Deglycosylation by small intestinal epithelial cell $\beta$-glucosidases is a critical step in the absorption and metabolism of dietary flavonoid glycosides in humans. Eur J Nutr 2003; 42: 29-42

43 Skalicka-Wozniak K, Glowniak K. Pressurized liquid extraction of coumarins from fruits of Heracleum leskowii with application of solvents with different polarity under increasing temperature. Molecules 2012; 17: 4133-4141

44 Waksmundzka-Hajnos M, Petruczynik A, Dragan A, Wianowska D, Dawidowicz AL. Effect of extraction method on the yield of furanocoumarins from fruits of Archangelica officinalis Hoffm. Phytochem Anal 2004; 15: 313-319

45 Walasek M, Grzegorczyk A, Malm A, Skalicka-Wozniak K. Bioactivityguided isolation of antimicrobial coumarins from Heracleum mantegazzianum Sommier \& Levier (Apiaceae) fruits by high-performance counter-current chromatography. Food Chem 2015; 186: 133-138

46 Li B, Zhang X, Wang J, Zhang L, Gao B, Shi S, Wang X, Li J, Tu P. Simultaneous characterisation of fifty coumarins from the roots of Angelica dahurica by off-line two-dimensional high-performance liquid chromatography coupled with electrospray ionisation tandem mass spectrometry. Phytochem Anal 2014; 25: 229-240

47 Liu M, Shi X, Yang W, Liu S, Wang N, Shi R, Qiao S, Wang Q, Wang Y. Quantitative analysis of nine coumarins in rat urine and bile after oral administration of Radix Glehniae extract by high-performance liquid chromatography-electrospray ionization tandem mass spectrometry. Biomed Chromatogr 2011; 25: 783-793

48 Prasain JK, Jones K, Brissie N, Moore R, Wyss JM, Barnes S. Identification of puerarin and its metabolites in rats by liquid chromatography-tandem mass spectrometry. J Agric Food Chem 2004; 52: 3708-3712

49 Prasain JK, Peng N, Moore R, Arabshahi A, Barnes S, Wyss JM. Tissue distribution of puerarin and its conjugated metabolites in rats assessed by liquid chromatography tandem mass spectrometry. Phytomedicine 2009; 16: 65-71

50 Martel S, Guillarme D, Henchoz Y, Galland A, Veuthey JL, Rudaz S, Carrupt $P A$. Chromatographic approaches for measuring log P. In: Mannhold R, editor. Drug properties: measurement and computation. Weinheim: Wiley-VCH; 2008: 331-356

51 Martel S, Gillerat F, Carosati E, Maiarelli D, Tetko IV, Mannhold R, Carrupt $P A$. Large, chemically diverse dataset of $\log P$ measurements for benchmarking studies. Eur J Pharm Sci 2013; 48: 21-29

52 Balimane PV, Pace E, Chong S, Zhu MS, Jemal M, Van Pelt CK. A novel high-throughput automated chip-based nanoelectrospray tandem mass spectrometric method for PAMPA sample analysis. J Pharm Biomed Anal 2005; 39: 8-16

53 Mensch J, Noppe M, Adriaensen J, Melis A, Mackie C, Augustijns P, Brewster ME. Novel generic UPLC/MS/MS method for high throughput analysis applied to permeability assessment in early Drug Discovery. J Chromatogr B Analyt Technol Biomed Life Sci 2007; 847: 182-187

54 Balimane PV, Chong S. Cell culture-based models for intestinal permeability: a critique. Drug Discov Today 2005; 10: 335-343 